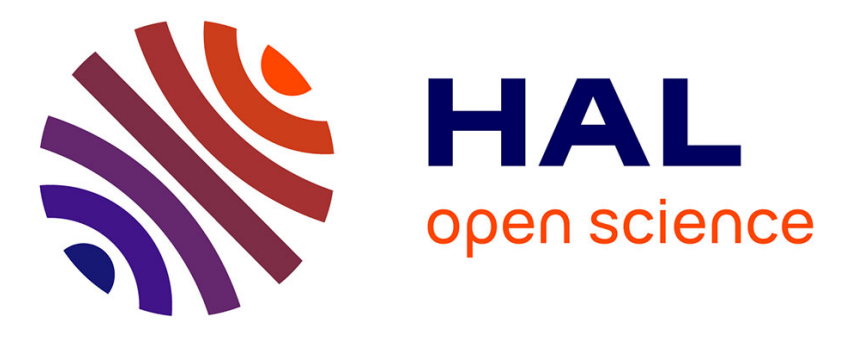

\title{
Deformation of silica glass studied by molecular dynamics: Structural origin of the anisotropy and non-Newtonian behavior
}

Xavier Bidault, Stéphane Chaussedent, Wilfried Blanc, Daniel R. Neuville

\section{- To cite this version:}

Xavier Bidault, Stéphane Chaussedent, Wilfried Blanc, Daniel R. Neuville. Deformation of silica glass studied by molecular dynamics: Structural origin of the anisotropy and non-Newtonian behavior. Journal of Non-Crystalline Solids, 2016, 10.1016/j.jnoncrysol.2015.11.029 hal-01351452

\section{HAL Id: hal-01351452 \\ https://hal.science/hal-01351452}

Submitted on 3 Aug 2016

HAL is a multi-disciplinary open access archive for the deposit and dissemination of scientific research documents, whether they are published or not. The documents may come from teaching and research institutions in France or abroad, or from public or private research centers.
L'archive ouverte pluridisciplinaire HAL, est destinée au dépôt et à la diffusion de documents scientifiques de niveau recherche, publiés ou non, émanant des établissements d'enseignement et de recherche français ou étrangers, des laboratoires publics ou privés. 


\title{
Deformation of silica glass studied by Molecular Dynamics: Structural origin of the anisotropy and non-Newtonian behavior
}

\author{
Xavier Bidault ${ }^{1, \mathbf{a}}$, Stéphane Chaussedent ${ }^{1}$, Wilfried Blanc ${ }^{2}$, Daniel R. Neuville ${ }^{3}$ \\ ${ }^{1}$ Université d'Angers, Laboratoire de Photonique d'Angers (LPhiA) - UPRES EA 4464, 2 Bd \\ Lavoisier 49045 ANGERS Cedex 01, France \\ ${ }^{2}$ Université Nice Sophia Antipolis, CNRS, Laboratoire de Physique de la Matière Condensée - \\ UMR 7336, Parc Valrose, 06100 Nice, France \\ ${ }^{3}$ Institut de Physique du Globe de Paris, Géomatériaux, CNRS, Sorbonne Paris Cité, 1 rue Jussieu, \\ 75005 PARIS, France
}

\begin{abstract}
A novel aspect of the medium-range structure of silica drawn into fibers is studied. The network of silica glass structure is composed of corner-shared $\mathrm{SiO}_{4}$ tetrahedra, and it can be seen as a structure of interconnected rings $(-\mathrm{Si}-\mathrm{O})_{\mathrm{n}}$ of various size, denoted nMR (n-Membered Ring). Molecular Dynamics simulations show that small-sized silica rings get a preferential orientation during the drawing, either during the high-temperature stage for $3 \mathrm{MR}$, or during the cooling for $4 \mathrm{MR}$ and $5 \mathrm{MR}$, and they persist in this state in the fiber at ambient temperature. This leads to a structural anisotropy, more specifically a "transverse isotropy", because of different longitudinal and transversal physical properties. This anisotropic structural rearrangement during the drawing process induces a non-Newtonian behavior of the modeled glass melt, with strain-rate dependent properties.
\end{abstract}

\section{Highlights:}

Anisotropy in silica glass comes from the orientation that small silica rings acquire during the deformation. The model is in agreement with experiments (non-Newtonian behavior of the melt, anisotropic elasticity of the fiber). The anisotropy in silica fiber is a "transverse isotropy".

Keywords: Silica glass, Molecular Dynamics, Glass structure, Anisotropy, Elasticity, non-Newtonian behavior.

\section{Introduction}

Glass is a ubiquitous material with many applications in daily life as well as in high technology, for instance in photonics or photovoltaic areas. To allow such versatility, the knowledge of glass structure is of prime importance. This glass structure has been subjected to many studies and many of them have been referenced by Jiang et al. [1]. Silica glass structure is described as a network of interconnected and randomly distributed $\mathrm{SiO}_{4}$ tetrahedra forming an isotropic material. However, Brückner [2] has evidenced quite a long time ago that anisotropies and orientations could be introduced into isotropic glass melts through non-Newtonian viscous deformations. A non-Newtonian behaviour is described by strain-rate dependant properties. This behaviour in silica-based glasses has been studied by Dingwell [3] \& Webb [4][5], to understand the rheology of magmas, and by Simmons et al. [6] and Li et al. [7] to address fiber manufacturing issues. It is associated by Simmons et al. [6] to structural rearrangements occurring in the material. In this way, a structural origin of these anisotropies is supposed to explain macroscopic property changes, such as the birefringence of E-glass (silica-based) fiber [8] or, much more recently, the different axial and radial elastic responses to an elastic mechanical stress in silica fiber [9].

Thus, the existence of anisotropy in silica fiber is a known fact, but despite many efforts, the topological nature of the involved structural rearrangement is still unknown. Many numerical simulations have been developed to study silica

\footnotetext{
${ }^{\mathrm{a}}$ Electronic mail: xavier.bidault@univ-angers.fr
} 
glass deformation, mostly at ambient temperature. So far, a bulk silica glass under tensile load has been modeled by Pedone et al. at low temperature to study the stress-strain behaviour [10]. Yuan et al. have studied parameters such as system size, cooling rate, strain rate and working temperature up to $1500 \mathrm{~K}$, to reproduce the experimental brittle fracture of amorphous silica nanowires [11]. Rountree et al. have simulated bulk silica glass under a simple shear at $300 \mathrm{~K}$ and have predicted a permanent plasticity-induced structural anisotropy [12], due to Si-O-Si directions remaining aligned with the strain/stress direction. An experimental radial X-ray diffraction study performed by Sato et al. [13] on silica glass after decompression has evidenced a persistent differential strain equivalent to a remaining differential stress. It has been explained by anisotropic reconstruction of inter-tetrahedra Si-O-Si bonds, therefore validating the above prediction.

In this article, we focus our attention on the drawing of a silica fiber at high temperature. Typically performed around $2300 \mathrm{~K}$, it is the common stage for most of the manufacturing processes to obtain an optical fiber. This crucial part has an important impact on its final properties such as refractive index profile or residual stress. The effects of various experimental conditions (drawing speed, tension, temperature, cooling profile) have been reported by Lancry $e t$ $a l$. [14]. Resulting fibers have various profiles of fictive temperature, which is known to describe the glass nanoscale structure. The structural modifications induced by this stage are investigated by means of Molecular Dynamics (MD) simulations. To our knowledge, this is the first MD simulation of the complete drawing process to obtain a fiber, in order to study the effects on silica glass. It appears that small silica rings get a persistent orientation in the fiber, leading to a structural anisotropy, which is called "transverse isotropy" because of different longitudinal and transversal physical properties. Moreover, the experimental link between this anisotropy and the non-Newtonian behaviour is verified in the simulations, since this anisotropic rearrangement leads to a non-Newtonian behaviour of the MD glass melt, with strain-rate dependant properties.

\section{Molecular Dynamics simulations}

\subsection{Silica glass modeling}

Classical molecular dynamics simulations are carried out by LAMMPS (Large-scale Atomic/Molecular Massively Parallel Simulator) [15] with periodic boundary conditions (PBC) and using the interatomic potential developed by Pedone et al. [16], designed to reproduce structural and mechanical properties of a wide range of silicates. This potential is composed of three terms: a long-range Coulomb interaction, a short-range Morse function and an additional $1 / r^{12}$ repulsive contribution. The long-range Coulomb term is evaluated using the Wolf method [17], consisting in a spherically-truncated, charge-neutralized, shifted, pairwise $1 / r$ summation. This method, initially developed for ionic crystals and melts, gives good results for amorphous materials like silica [18][19], and silica-based [20] glasses. It also well reproduces the energetics and the dynamics of various typical systems [21]. The main advantages of the Wolf method are: (a) the computational cost increases linearly with the system size $N$, instead of, at best, $N^{3 / 2}$ for Ewald method ( $N$ is the number of atoms involved in the simulation); (b) the inherent periodicity of the Ewald sum is no more imposed to the system. The parameters of the Wolf method are chosen among the tested ones giving the best match between Wolf and Ewald summations, by comparing the total energy and the short- and medium-range structures obtained after melt/quench sequences of a pure $\mathrm{SiO}_{2}$ system. Hence, the long-range cutoff is set at $7.5 \AA$ and the damping parameter is set at $0.30 \AA^{-1}$. For this study, the short-range cutoff of the Morse function is set at $5.5 \AA$.

The usual melt/quench technique is used to model a glass from an initial crystalline structure. Unless otherwise indicated, the simulations are made at constant atmospheric pressure ( $\mathrm{P}=1$ bar), using the Nosé-Hoover NPT algorithm (constant Number of atoms, controlled Pressure and Temperature) and a time step of $1 \mathrm{fs}$. The starting configuration, melted at $4000 \mathrm{~K}$, is continuously cooled down at $5 \mathrm{~K} / \mathrm{ps}$, and finally equilibrated at $300 \mathrm{~K}$. Despite a cooling rate many orders faster than in a laboratory, a common choice around $5 \mathrm{~K} / \mathrm{ps}$ yields the expected structure when compared to experimental data. More specifically, the structure of a silica glass modeled with Pedone potential and the structure derived from experimental neutron diffraction are both in very good agreement [22]. This model of silica glass has a density of $2.28 \mathrm{~g} / \mathrm{cm}^{3}$ and a glass transition temperature $\mathrm{T}_{\mathrm{g}}$ of $2620 \pm 40 \mathrm{~K}$. This density, $3.6 \%$ higher than experimental value of $2.20 \mathrm{~g} / \mathrm{cm}^{3}$, is in agreement with the anomalous behavior of silica glass observed by Brückner [23] and Shelby [24]. Indeed, the slower the quench rate is, the lower the experimental density of silica glass is. MD quench rate being many orders higher than laboratory ones, it seems consistent for the model to have a higher density than experimental one. This anomalous behavior of silica glass also appears in the MD simulations made by Vollmayr et al. [25], using the BKS potential, and it has also been verified for the potential used in this paper for various quench rates. Experimental $\mathrm{T}_{\mathrm{g}}$ is estimated to be $1446 \mathrm{~K}$ [26] (defined as the temperature when the viscosity is $10^{12}$ Pa.s) or 
$1473 \mathrm{~K}$ [27] (determined by differential scanning calorimetry at $0.33 \mathrm{~K} / \mathrm{s}$ ). Whatever the type of potential used in MD simulations, the overestimation of $\mathrm{T}_{\mathrm{g}}$ is a known problem [25][28], which however does not impact the structural properties of the glass. Therefore, as indicated hereafter, the drawing simulation is performed using this modeled glass as the preform.

\subsection{High-temperature drawing process}

Experimentally, a silica preform placed on the top of a draw tower is heated between $2200 \mathrm{~K}$ and $2600 \mathrm{~K}$, which is over its softening temperature of $1900 \mathrm{~K}$, which is higher than experimental $\mathrm{T}_{\mathrm{g}}$ of approximately $1450 \mathrm{~K}$. According to Urbain et al. [29], this temperature range corresponds to working viscosities between $10^{3}$ and $10^{5}$ Pa.s. Fused silica flows down with gravity, forming a fiber which is drawn down to maintain the process. The MD simulation, schematized in Figure 1, consists in modeling the high-temperature uniaxial deformation and the cooling until complete relaxation of a small volume of silica localized at the center of the preform, along its axis. The starting simulation box, of approximately 300x50x300 $\AA^{3}$, contains 314928 atoms of our modeled silica glass. This box is heated at $2700 \mathrm{~K}$ and 1 bar, and equilibrated. This temperature choice is guided by the real process, where the deformation temperature is higher than the $\mathrm{T}_{\mathrm{g}}$. The uniaxial drawing is made by an anisotropic NPT algorithm reproducing a natural deformation along $y$-axis with a "constant true strain rate": the height evolves as $L_{y}(t)=L_{y 0} e^{v t}$ with a "true strain rate" (TSR), also denoted $v$, of $10^{9} \mathrm{~s}^{-1}$, while no stress is imposed to lateral sides (only interactions due to atoms in periodic-boundarycondition replicas of the box). After $3500 \mathrm{ps}$, the box volume is approximately 50x1800x50 $\AA^{3}$. It is now considered to be outside the furnace and it must be cooled down. Experimental cooling procedures can be either passive in a tall draw tower, or forced when high draw speeds are needed [30], or even controlled with specific temperature profiles leading to industrial patents. In this simulation, the box undergoes simultaneously and progressively for 300 ps a release of the uniaxial stress $\sigma_{y}$ and a decrease of the temperature to reach 1 bar and $300 \mathrm{~K}$, respectively, and it is finally equilibrated at $300 \mathrm{~K}$. The resulting fiber density is $2.22 \mathrm{~g} / \mathrm{cm}^{3}$. No fracture is observed. Annealing is out of the scope of this paper.

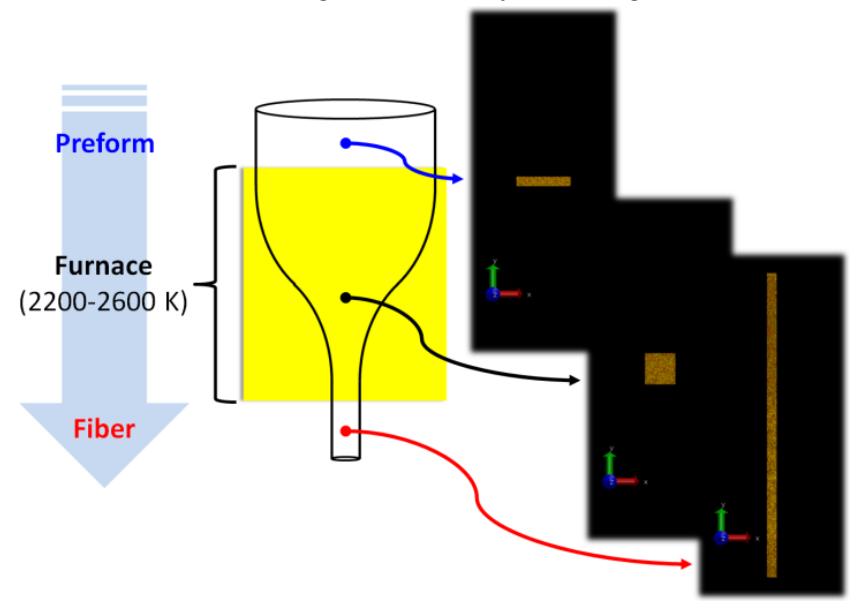

Fig. 1. Laboratory drawing protocol. Rendering of three steps of the MD simulation: initial (preform), during the hightemperature drawing, and final (fiber).

At a larger scale, assuming an axisymmetric 2D laminar flow in a cylindrical system, the use of a fluid dynamic model with volume discretization leads to a deformation of the whole preform more complex [31] than a simple elongation. But the size of the used elements, at least $4 \mu \mathrm{m}$ in the radial direction, is far bigger than the size of our simulation box. Hence, a simple elongation remains a very good approximation at the nanoscale level.

The choice of $50 \AA$ as initial and final smallest dimension is a minimum length to proceed with ring statistics up to 14-membered silica rings. Other initial and final dimensions are imposed by the strain rate as well as computer resources. Pedone et al. use "engineer strain rates" (ESR) of $10^{8}$ and $10^{9} \mathrm{~s}^{-1}$ to study silica glass under tensile load at $300 \mathrm{~K}[10]$. Thus, a TSR $v$ of $10^{9} \mathrm{~s}^{-1}$ is appropriate to study the drawing just above glass transition. The very small stress overshoot $(7 \%)$ between elastic and plastic regions of the stress-versus-strain curve displayed in Figure 2 confirms that the chosen TSR is slow enough to allow molecular restructuration rather than breakdown of glass structure [6]. During the plastic deformation, the stress remains practically constant at $1.5 \mathrm{GPa}$ and enables the calculation of the apparent viscosity [6] $\eta=\sigma_{y} / 3 v$, which is equal to $0.50 \mathrm{~Pa}$.s. This value is normally too low for molten silica to support a laboratory strain rate and such a material would be too liquid to form a fiber. However, the 
MD strain rate is many orders higher than laboratory ones and the stress-vs-strain curve confirms that the MD material supports this deformation without any fracture, and hence that it is able to form a fiber while undergoing a viscous flow stress. The apparent viscosity $\eta$ is compared to the viscosity $\eta_{0}$ of the unstressed MD material (i.e. the preform) at the same temperature. $\eta_{0}$ is computed according to the Green-Kubo methodology [32], and is found to be equal to $0.7 \mathrm{~Pa} . \mathrm{S}$ at $2700 \mathrm{~K}$. The apparent viscosity $\eta$ is lower than $\eta_{0}$. This indicates that the modeled high-temperature drawing corresponds to a non-Newtonian viscous flow [6], and thus that some of the fiber properties are strain-rate dependent. This aspect is presented in more detail in the paragraph 3.4.

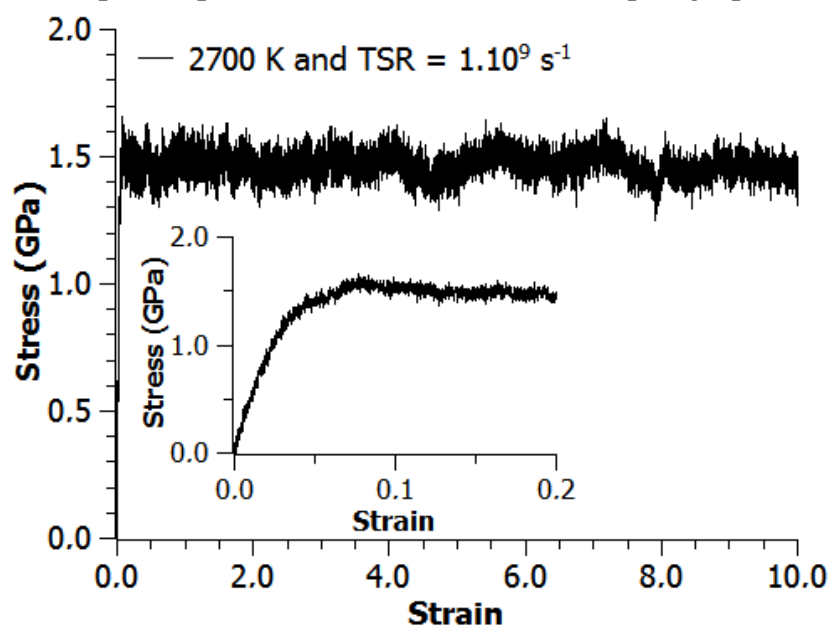

Fig. 2. Stress versus strain during the drawing process. Stress during the plastic deformation is around $1.5 \mathrm{GPa}$. Inset: elastic region followed by a very small overshoot $(7 \%)$.

One can note that, using the potential of Pedone et al. [16], MD viscosities are in any case two orders of magnitude below the experimental viscosity of $335 \mathrm{~Pa} . \mathrm{s}$ reported at $2755 \mathrm{~K}$ by Urbain et al. [29]. If a potential like the BKS one yields a viscosity only one order below experimental one [33], the problem is that the $\mathrm{T}_{\mathrm{g}}$ of this BKS silica glass is much more overestimated than the $\mathrm{T}_{\mathrm{g}}$ of silica glass modeled with Pedone potential (see paragraph 2.1). Indeed, in the same conditions, BKS silica glass is found to have a $\mathrm{T}_{\mathrm{g}}$ of $3130 \mathrm{~K}$ and its Green-Kubo viscosity $\eta_{0}$ at $3200 \mathrm{~K}$ (just above glass transition) is 1.2 Pa.s. Hence, whatever the potential, the MD drawing just above glass transition is carried out at the same viscosity magnitude. A high-temperature drawing of silica glass with BKS potential has been performed at $3200 \mathrm{~K}$ with a TSR of $10^{9} \mathrm{~s}^{-1}$, and it has been found to give similar results to the ones obtained hereafter with Pedone potential.

The strain $\varepsilon(t)=e^{v t}-1$, is about 36 at the end of the simulation, resulting in a ratio of 6 for lateral dimensions. It is far from the preform-to-fiber diameter ratio usually obtained in a laboratory, which is around 80 . To achieve such a value in MD, initial lateral sides should be 14 times larger, involving almost 200 times more atoms during approximately 9000 ps. This is far beyond our current computer resources. However, we presuppose that our statistics give the right trend.

\section{Results}

\subsection{Ring structure of silica glass}

$\mathrm{SiO}_{4}$ tetrahedron (one Silicon atom surrounded by four Oxygen atoms) is the main structural unit composing silica glass. These tetrahedra are linked by corner-shared Oxygen to form rings ( $\mathrm{Si}-\mathrm{O})_{\mathrm{n}}$ denoted nMR (n-Membered-Ring), with $\mathrm{n}$ typically ranging from 3 to 11 . These rings are interconnected (a simple example with two rings is given in Figure $3 b$ ) to form a 3-dimensional ring structure. In order to analyze this atomic medium-range structure, the ring size distribution is computed with Yuan \& Cormack algorithm [34]. The Figure 4 reports these distributions for modeled preform and fiber. Currently, an experimental distribution in 3D silica glass has not yet been found, but there exist some for 2D glass, determined by scanning tunneling microscopy (STM) [35] [36]. Heyde et al. [36] propose a log-normal shape for this distribution. A fit of their cumulative distribution (with a correlation coefficient $\mathrm{R}^{2}$ of 0.9989 ) estimates that 3 -membered rings (3MR) represent $0.12 \%$ of the total number of rings. In the modeled preform, the $14063 \mathrm{MR}$ represent $0.65 \%$ of the total number of rings. Interestingly, this value is not so far from the extrapolated experimental data. In modeled fiber, the $16853 \mathrm{MR}$ represent $0.80 \%$ of the total number of rings. 3MR are more numerous in the 
fiber than in the preform (by about $20 \%$ in our model). This is consistent with the Raman study of Alessi et al. [37], and this trend is also observed for the $4 \mathrm{MR}$.
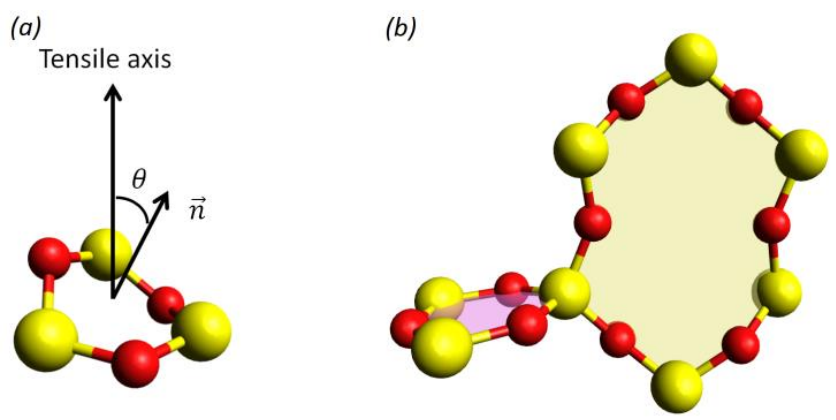

Fig. 3. Silica rings. (a) Unit vector $\vec{n}$ normal to the mean surface of a 3-membered ring (3MR) (yellow Silicon and red Oxygen - color online); (b) Representative case of a 3MR linked to a 6MR.

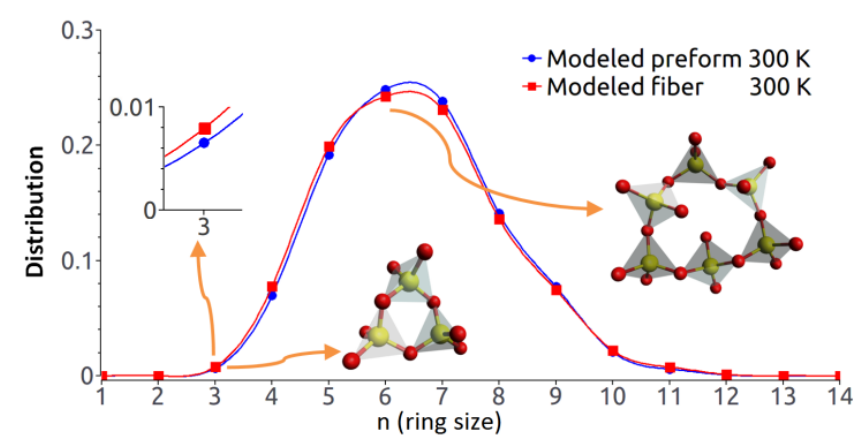

Fig. 4. Ring size distributions in modeled silica-glass preform and fiber. Drawing performed at $2700 \mathrm{~K}$ with a strain rate of $10^{9} \mathrm{~s}^{-1}$. Dots are connected by an arbitrary curve.

\subsection{Structural anisotropy due to small-sized rings}

To our knowledge, the orientation of silica rings was only studied by Ghemid et al. [38]. Taking into account that they studied a uniaxial compression (whereas a uniaxial traction is studied here) and despite poor statistics, their conclusion about the rings of the smallest size is consistent with ours. However, implication for anisotropic macroscopic properties is only supposed. Here, we propose a study with better statistics, especially for the notnumerous small rings, with extended conclusions. For each ring, the mean-plane equation is determined by least-square method, yielding the components of the normal-to-the-ring unit vector $\vec{n}$, with $\theta \in\left[0^{\circ}, 90^{\circ}\right]$ as schematized in Figure $3 a$. Finally, a scalar value $n_{y} \in[0,1]$ is obtained through the projection of $\vec{n}$ on the tensile axis (here the $y$-axis). For instance, $n_{y}=1$ means that the ring mean surface is perpendicular to the tensile axis. An important property of this projection is its uniform distribution for an isotropic material.

Before going any further, we want to mention that the monitoring of one specific ring during the whole process of the simulation is not possible. While the simulation box deforms, rings are continuously made and unmade as silica tetrahedra move. For example, because of its very short lifetime, a 3MR can rarely be tracked over 20 ps (approximately $0.5 \%$ of the total simulation time). This is consistent with the study of a silica zeolite where $3 \mathrm{MR}$ are found to be the most unstable [39]. Thus, this study deals with the evolution of successive statistical sets, and ringorientation distributions during the drawing process are represented in Figure $5 a$, for $3 \mathrm{MR}, 4 \mathrm{MR}, 5 \mathrm{MR}$ and $6 \mathrm{MR}$. In this figure, the projection $n_{y}$ of $\vec{n}$ on tensile axis is simply discriminated as below or above 0.5 , meaning that the ring mean surface has a trend to be parallel or perpendicular to the stress axis, respectively. The more tilted the segment is, the more anisotropy there is.

A uniform orientation distribution is verified for the rings of each size in the preform at $300 \mathrm{~K}$. The slight $0.5 \%$ deviation to uniformity for the $3 \mathrm{MR}$ and $6 \mathrm{MR}$ is explained by the quite low number of the former (1406 3MR, representing $0.65 \%$ of the total number of rings), and the various shapes the latter can have. While the drawing at 2700 $\mathrm{K}$ is performed, $3 \mathrm{MR}$ behave as the most reactive to the traction. Their mean surface tends to orient perpendicular to the stress axis and they keep this orientation until the end of the high-temperature stage. During the relaxation, as the stress and the temperature decrease, this induced orientation becomes less pronounced but remains persistent in the fiber at 
300 K. 4MR and 5MR do not seem to be affected during the high-temperature stage, but they do during the relaxation and they also get a persistent orientation with a perpendicular-to-stress-axis trend in the fiber at $300 \mathrm{~K}$. Unlike others, $6 \mathrm{MR}$ tend to have their mean surface parallel to stress axis at $2700 \mathrm{~K}$, and to be fully relaxed at $300 \mathrm{~K}$ with a quasiuniform orientation distribution. Consequently, there is a structural anisotropy in the modeled fiber due to the persistent orientation of small rings: $3 \mathrm{MR}, 4 \mathrm{MR}$ and $5 \mathrm{MR}$.
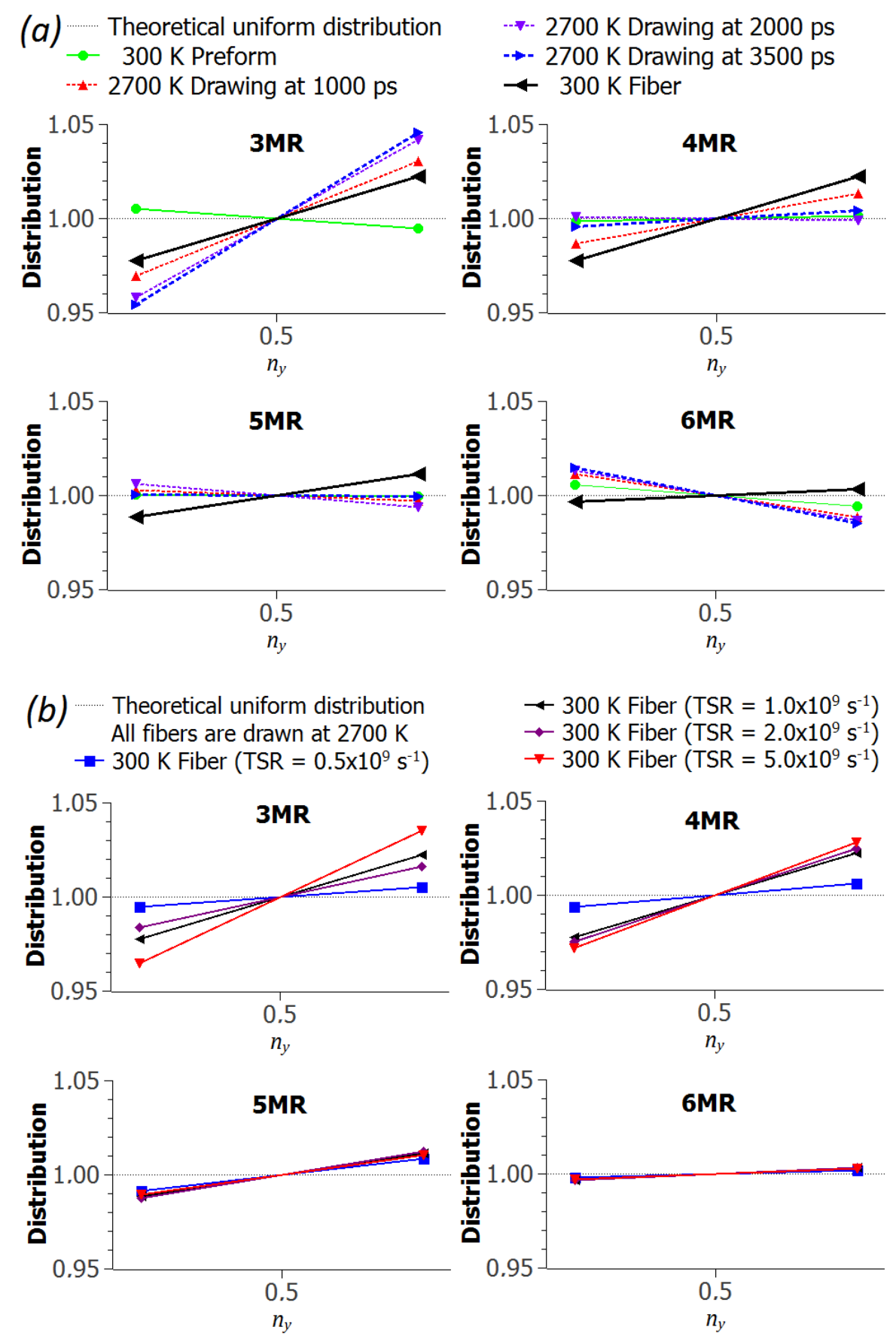

Fig. 5. Ring orientations induced by the drawing process (color online). (a) During the drawing at $2700 \mathrm{~K}$ with a strain rate of $10^{9} \mathrm{~s}^{-1}$; (b) Influence of strain rate (TSR) on ring orientation in $300 \mathrm{~K}$ fibers drawn at $2700 \mathrm{~K}$. The projection $n_{y}$ of $\vec{n}$ on tensile axis is simply discriminated as "below" or "above" 0.5 , respectively meaning a ring mean surface with a "parallel-" or a "perpendicular-" to-stress-axis trend. The more tilted the segment is, the more anisotropy there is.

\subsection{Elastic anisotropy of the fiber}

A way to quantify this anisotropy is to compute and compare the components $C_{i j}$ of the elastic constant matrices $C$ of the modeled preform and fiber. Both of them are forced to deform with a small strain $\varepsilon_{j}$ of $10^{-3}$. After energy 
minimization, the induced stresses $\sigma_{i}$ are computed and allow the $C_{i j}$ determination with the Hooke's law $\sigma_{i}=C_{i j} \varepsilon_{j}(i$ and $j$ from 1 to 6 , according to the Voigt notation). Young's moduli $\mathrm{E}_{\mathrm{x}}, \mathrm{E}_{\mathrm{y}}$ and $\mathrm{E}_{\mathrm{z}}$ are calculated from the elastic compliance matrix $S=C^{-1}$, using $\mathrm{E}_{\mathrm{x}}=S_{11}^{-1}, \mathrm{E}_{\mathrm{y}}=S_{22}^{-1}$ and $\mathrm{E}_{\mathrm{z}}=S_{33}^{-1}$. Results are presented in the Table 1 .

For a bulk silica glass having a density of $2.20 \mathrm{~g} / \mathrm{cm}^{3}$, the experimental value of its Young's modulus is 72.5 GPa [40]. Fraser [41] has measured transverse and longitudinal sound velocities in silica glasses according to their densities. This allows an experimental determination of the variation of the Young's modulus E with the density: regardless of the silica glass origin, the more densified it is, the higher its Young's modulus is. The Young's moduli of our modeled preform and fiber are higher than the experimental value of $72.5 \mathrm{GPa}$ at $2.20 \mathrm{~g} / \mathrm{cm}^{3}$, but our models also have higher densities: $2.28 \mathrm{~g} / \mathrm{cm}^{3}$ and $2.22 \mathrm{~g} / \mathrm{cm}^{3}$, respectively. Pedone et al. [22] have modeled a silica glass of 2.20 $\mathrm{g} / \mathrm{cm}^{3}$ and its Young modulus is $72.1 \mathrm{GPa}$, very close to the experimental value. Since Yuan \& Huang [11] have evidenced that the short-range cutoff of the repulsive part of the potential has an influence on silica-glass elastic properties and density, it is worth mentioning that we have compared glasses modeled with the same short-range cutoff, of $5.5 \AA$ for the Morse function of this potential. Hence, the behavior of the modeled silica glass is as expected: the denser it is, the higher its Young's modulus is.

The preform matrix has the typical form of an isotropic material, with only two independent components: non-zero components can be determined from the shear modulus $G=33.3 \mathrm{GPa}$ and the bulk modulus $K=46.5 \mathrm{GPa}$ with $C_{11}=C_{22}=C_{33}=K+4 G / 3, C_{12}=C_{13}=C_{23}=K-2 G / 3$ and $C_{44}=C_{55}=C_{66}=G$. Comparatively, the fiber matrix has the typical form of an anisotropic material presenting a "transverse isotropy", with a uniaxial symmetry (along $y$-axis) and five independent components: $C_{11}=C_{33}, C_{12}=C_{23}, C_{13}, C_{22}, C_{44}=C_{66}$. Regarding $C_{55}$, it equals $\left(C_{11}-C_{13}\right) / 2$. This anisotropy is confirmed by the Young's moduli of the fiber, with $\mathrm{E}_{\mathrm{y}}$ that clearly differs from $\mathrm{E}_{\mathrm{x}}$ and Ez. This elastic anisotropy in silica fiber has been evidenced very recently by Champagnon et al. [9], who have measured a difference between the axial and the radial Brillouin strain coefficients.

Table 1. Elastic constants $C_{i j}(\mathrm{GPa})$ of modeled preform and fiber (Voigt notation), and Young's moduli $\mathrm{E}_{\mathrm{x}}, \mathrm{E}_{\mathrm{y}}$ and $\mathrm{E}_{\mathrm{z}}$

\begin{tabular}{|c|c|c|c|c|c|c|c|c|c|c|c|c|}
\hline & \multicolumn{6}{|c|}{ Preform } & \multicolumn{6}{|c|}{ Fiber } \\
\hline & 90.7 & 24.2 & 24.3 & 0.1 & 0.2 & -0.3 & 84.2 & 22.3 & $21.5^{\mathrm{a}}$ & -0.1 & 0.1 & 0.1 \\
\hline & & 91.2 & 24.4 & -0.1 & -0.1 & -0.2 & & $87.0^{\mathrm{a}}$ & 22.7 & -0.1 & 0.0 & -0.2 \\
\hline$C_{i j}$ & & & 90.6 & 0.2 & 0.3 & -0.1 & & & 85.4 & -0.3 & 0.1 & -0.0 \\
\hline$(\mathrm{GPa})$ & & & & 33.4 & 0.0 & -0.0 & & & & 31.8 & -0.1 & 0.1 \\
\hline & & & & & 33.3 & 0.0 & & & & & $31.7^{\mathrm{a}}$ & -0.1 \\
\hline & & & & & & 33.3 & & & & & & 31.8 \\
\hline $\begin{array}{c}\mathrm{E}_{\mathrm{x}}, \mathrm{E}_{\mathrm{y}}, \mathrm{E}_{\mathrm{z}} \\
(\mathrm{GPa})\end{array}$ & & & & 80.5 & 80.9 & 80.3 & & & & 75.4 & $77.5^{b}$ & 76.3 \\
\hline
\end{tabular}

${ }^{\mathrm{a}} C_{22}$ differs from $C_{11}$ and $C_{33}, C_{13}$ differs from $C_{12}$ and $C_{23}$, and $C_{55}$ differs from $C_{44}$ and $C_{66}$, meaning a transverse isotropy around $y$-axis in fiber.

${ }^{b} \mathrm{E}_{\mathrm{y}}$ differs from $\mathrm{E}_{\mathrm{x}}$ and $\mathrm{E}_{\mathrm{z}}$.

\subsection{Effect of strain rate on anisotropy and implication for non-Newtonian behavior}

A stress-induced anisotropy can be observed as birefringence in glass melts deformed with high strain rates [2][8]. Simmons et al. [6], who have performed elongation experiments on soda-lime silica glass fibers, have evidenced that such high strain rates lead to a non-Newtonian behavior: the apparent viscosity [6] $\eta=\sigma / 3 v$ deviates from its Newtonian value $\eta_{0}$, and $\eta$ becomes strain-rate dependent. This dependence also appears in our MD simulations performed at $2700 \mathrm{~K}$ and at different strain rates. Figures $6 a$ and $6 b$ report the evolution of the uniaxial stress $\sigma$ and the apparent viscosity $\eta$ with imposed strain rate $v$. Similarities with graphical representations of experimental data from Simmons et al. [6] are very interesting. At high strain rate, the stress deviates from its asymptote of slope $3 \eta_{0}$, and the apparent viscosity effectively becomes lower than $\eta_{0}$. The effects of these different strain rates (TSR) on ring orientation (i.e. on anisotropy) in $300 \mathrm{~K}$ fibers drawn at $2700 \mathrm{~K}$ are represented in Figure $5 b$. Whatever the strain rate, $6 \mathrm{MR}$ are not affected, and 5MR have quite the same level of orientation. At the slowest strain rate $\left(0.5 \times 10^{9} \mathrm{~s}^{-1}\right)$, induced orientations of $3 \mathrm{MR}, 4 \mathrm{MR}$ and $5 \mathrm{MR}$ are low, resulting in a low anisotropy. This is consistent with the uniaxial stress very close to its asymptotic behavior, and with the apparent viscosity close to its Newtonian value. But at higher strain rates, the $3 \mathrm{MR}$ and $4 \mathrm{MR}$ trend to be perpendicular to stress axis is enhanced, which also enhances the anisotropy. In 
turn, this accentuated structural anisotropy, which is clearly strain-rate dependent, enhances the non-Newtonian behavior of the MD glass melt, with a decreasing viscosity.
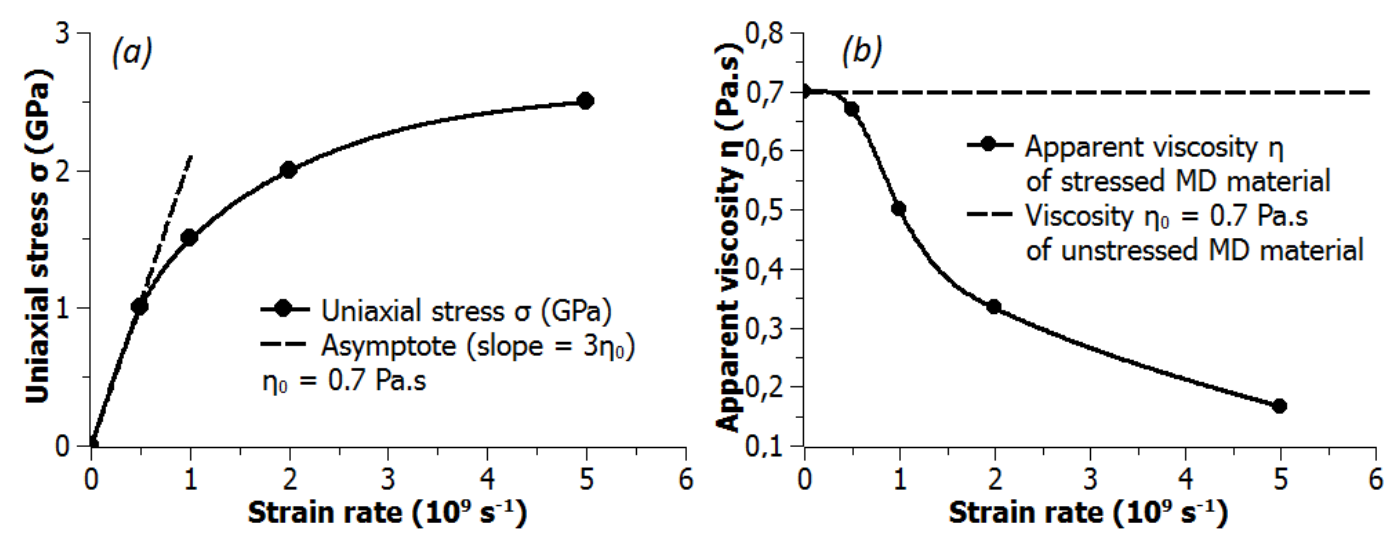

Fig. 6. Non-Newtonian behavior of the glass melt. Evolution of (a) uniaxial stress $\sigma$, and $(b)$ apparent viscosity $\eta$, with imposed strain rate $v$.

\section{Discussion}

\subsection{Origin of the different $3 M R$ orientation}

When a uniaxial stress is applied, rings behave differently according to their size. This mainly depends on their ability to deform. The study of the Young modulus evolution according to the monomer ring size of silica nanotubes undergoing stretching or bending [42] indicates that the more members the ring has, the more flexible it is. So, before breaking, a large ring can stretch along the stress axis while a small ring cannot. Ring statistics based on either scanning tunneling microscopy (STM) of 2D silica glass [35] [36] or MD simulations of 3D silica glass [25] [43] both show an asymmetric size distribution around 6MR (see also Figure 4). Consequently, the probability for a $3 \mathrm{MR}$ to connect to a $6 \mathrm{MR}$ is very high. In our simulation, $80 \%$ of $\mathrm{Si}$ atoms belonging to a $3 \mathrm{MR}$ also belong to a $6 \mathrm{MR}$. A representative case schematized in Figure $3 b$ helps to understand why a 3MR tends to be perpendicular to the stress axis at $2700 \mathrm{~K}$. Due to the tetrahedral geometry of the linking Silicon, their mean surfaces are perpendicular to each other. Since a $6 \mathrm{MR}$ tends to orient its surface parallel to the stress axis during the high-temperature stage, then a $3 \mathrm{MR}$ tends to be perpendicular to the drawing direction. The behavior during the cooling is more difficult to interpret. Some of the $6 \mathrm{MR}$ connected to a $3 \mathrm{MR}$ can remain aligned with the former stress axis, explaining why the 3MR still have an orientation trend. But $6 \mathrm{MR}$ connected to $3 \mathrm{MR}$ are not numerous (only $4.4 \%$ of $\mathrm{Si}$ atoms belonging to a $6 \mathrm{MR}$ also belong to a $3 \mathrm{MR}$ ). This low contribution to $6 \mathrm{MR}$ orientation distribution may be hidden in the quasi-uniformity that $6 \mathrm{MR}$ recover at $300 \mathrm{~K}$, and by the newly formed 6MR during the cooling that are likely to have a quasi-uniform orientation distribution due to the absence of stress axis.

\subsection{Exclusion of a possible density anisotropy}

To ensure that the elastic anisotropy of the fiber is not due to a preferred packing of the atoms along or perpendicular to the stress axis, the density distributions of the fiber seen under various directions has been determined. The modeled fiber has been sliced up with a thickness of $5 \AA$ along $x$-, $y$ - or $z$-axis, yielding the density distributions $\rho_{x}, \rho_{y}$, and $\rho_{z}$, respectively. The mean values of $\rho_{x}, \rho_{y}$, and $\rho_{z}$ are the same, within $\pm 0.001 \mathrm{~g} / \mathrm{cm}^{3}$. Various slice thicknesses have been tested, yielding the same tiny deviation, whose sign is not correlated to slicing direction. The same result is obtained from each modeled fiber, whatever the strain rate at which it has been drawn. Hence, there is no density anisotropy.

\subsection{Exclusion of a possible orientation anisotropy of Si-O-Si bonds}

Another possible structural origin of the fiber anisotropy is investigated, by using the fabric tensor as defined by Rountree et al. [12]. It allows the determination of the mean orientation of Si-O-Si bonds. From the eigenvalues of this fabric tensor, they define an anisotropy index $\alpha$ ranging from 0 (isotropic material) to 1 (fully anisotropic material). For 
instance, the calculated value $\alpha=0.03$ describes the anisotropy of the crystalline structure of $\alpha$-Quartz. A value of $\alpha$ around $10^{-3}$ describes an isotropic silica glass, or the recovered structure after unloading of a glass deformed within its elastic domain [12]. Comparatively, the anisotropy index of our modeled fibers ranges between $0.8 \times 10^{-3}$ and $1.6 \times 10^{-3}$, without any correlation to the strain rate at which they have been drawn. This result is in contrast with the anisotropy induced by a shear deformation within the plastic domain at $300 \mathrm{~K}$. Indeed, Rountree et al. have evidenced a persistent $\mathrm{Si}-\mathrm{O}-\mathrm{Si}$ orientation and this is not the case in our modeled fibers. Interestingly, it seems that anisotropies induced by a shear deformation at ambient temperature or by an elongation at high-temperature have different origins.

Finally, the anisotropy index, now extracted from the fabric tensor used to determine the mean orientation of Si-O bonds, leads to the same conclusion. Its value ranges between $1.5 \times 10^{-3}$ and $2.1 \times 10^{-3}$, without any correlation to the strain rate at which the fiber has been drawn. All these structural considerations confirm the only implication of $3 \mathrm{MR}$, 4MR and 5MR for the anisotropy induced by high-temperature elongation of silica-glass to form a fiber.

\section{Conclusion}

Experimentally, the anisotropy in fibers has already been stated in previous studies, for instance by Brückner [2] and Stockhorst [8] for silica-based fibers, and by Champagnon et al. [9] for silica fibers. The existence of this anisotropy in silica fibers is confirmed once more in this study, by means of Molecular Dynamics simulations, which clearly establish the structural origin of this anisotropy. It comes from the persistent orientation that small rings acquire during the deformation (during the high-temperature stage for the $3 \mathrm{MR}$, and during the release of the uniaxial stress and the temperature decrease for the 4MR and $5 \mathrm{MR}$ ). The different axial and radial elastic properties of the modeled fiber (as well as the real fiber [9]) indicates that this anisotropy is a "transverse isotropy". Moreover, the higher the strain rate of the deformation is, the more effective the anisotropy is, and the more non-Newtonian the glass melt becomes (with strain-rate dependent properties). Despite some limitations of the model used in this study (low viscosity and overestimated glass transition temperature), the use of a high strain rate allows mimicking the silica glass behavior, as observed experimentally.

To emphasize the results presented in this paper and to complete existing experimental data, other quantitative ones could be obtained from optical or elastic properties. The cross-section index profile of a fiber could be determined by transverse multiple-beam interferometry [44], and compared to a longitudinal measure of it. Longitudinal and transversal elastic properties could be accessed by acoustic contact-resonance AFM [45] [46] [47] [48] . Finally, this study may also benefit the field of optical fiber nanowires and microwires [49], manufactured from the tapering of a fiber at high-temperature.

\section{Acknowledgements}

This work was supported by grants from Région Pays de la Loire (NACRYSIM program), GDR Verres, and by ANR project "Nice-DREAM". This research used resources of the CCIPL (Centre de Calcul Intensif des Pays de Loire).

\section{References}

[1] Z.-H. Jiang and Q.-Y. Zhang, Prog. Mater. Sci. 61, 144 (2014).

[2] R. Brückner, J. Non-Cryst. Solids 95-96, Part 2, 961 (1987).

[3] D. B. Dingwell and S. L. Webb, Phys. Chem. Miner. 16, 508 (1989).

[4] S. L. Webb and D. B. Dingwell, J. Geophys. Res. Solid Earth 95, 15695 (1990).

[5] S. L. Webb and D. B. Dingwell, Phys. Chem. Miner. 17, 125 (1990).

[6] J. H. Simmons, R. K. Mohr, and C. J. Montrose, J. Appl. Phys. 53, 4075 (1982).

[7] J. H. Li and D. R. Uhlmann, J. Non-Cryst. Solids 3, 127 (1970).

[8] H. Stockhorst and R. Brückner, J. Non-Cryst. Solids 49, 471 (1982).

[9] B. Champagnon, S. Degioanni, and C. Martinet, J. Appl. Phys. 116, 123509 (2014).

[10] A. Pedone, G. Malavasi, M. C. Menziani, U. Segre, and A. N. Cormack, Chem. Mater. 20, 4356 (2008).

[11] F. Yuan and L. Huang, J. Non-Cryst. Solids 358, 3481 (2012).

[12] C. L. Rountree, D. Vandembroucq, M. Talamali, E. Bouchaud, and S. Roux, Phys. Rev. Lett. 102, 195501 (2009).

[13] T. Sato, N. Funamori, and T. Yagi, J. Appl. Phys. 114, 103509 (2013).

[14] M. Lancry, E. Régnier, and B. Poumellec, Prog. Mater. Sci. 57, 63 (2012).

[15] S. Plimpton, J. Comput. Phys. 117, 1 (1995). 
[16] A. Pedone, G. Malavasi, M. C. Menziani, A. N. Cormack, and U. Segre, J. Phys. Chem. B 110, 11780 (2006).

[17] D. Wolf, P. Keblinski, S. R. Phillpot, and J. Eggebrecht, J. Chem. Phys. 110, 8254 (1999).

[18] A. Carré, L. Berthier, J. Horbach, S. Ispas, and W. Kob, J. Chem. Phys. 127, 114512 (2007).

[19] P. Brommer, P. Beck, A. Chatzopoulos, F. Gähler, J. Roth, and H.-R. Trebin, J. Chem. Phys. 132, 194109 (2010).

[20] P. Demontis, S. Spanu, and G. B. Suffritti, J. Chem. Phys. 114, 7980 (2001).

[21] C. J. Fennell and J. D. Gezelter, J. Chem. Phys. 124, 234104 (2006).

[22] A. Pedone, G. Malavasi, A. N. Cormack, U. Segre, and M. C. Menziani, Chem. Mater. 19, 3144 (2007).

[23] R. Brückner, J. Non-Cryst. Solids 5, 123 (1970).

[24] J. E. Shelby, J. Non-Cryst. Solids 349, 331 (2004).

[25] K. Vollmayr, W. Kob, and K. Binder, Phys. Rev. B 54, 15808 (1996).

[26] C. A. Angell, J. Phys. Chem. Solids 49, 863 (1988).

[27] C. A. Angell, J. Non-Cryst. Solids 354, 4703 (2008).

[28] T. F. Soules, G. H. Gilmer, M. J. Matthews, J. S. Stolken, and M. D. Feit, J. Non-Cryst. Solids 357, 1564 (2011).

[29] G. Urbain, Y. Bottinga, and P. Richet, Geochim. Cosmochim. Acta 46, 1061 (1982).

[30] T. Vaskopulos, C. Polymeropoulos, and A. Zebib, Int. J. Heat Mass Transf. 38, 1933 (1995).

[31] Z. Wei, K.-M. Lee, S. W. Tchikanda, Z. Zhou, and S.-P. Hong, J. Heat Transf. 126, 713 (2004).

[32] R. E. Jones and K. K. Mandadapu, J. Chem. Phys. 136, 154102 (2012).

[33] J. Horbach and W. Kob, Phys. Rev. B 60, 3169 (1999).

[34] X. Yuan and A. N. Cormack, Comput. Mater. Sci. 24, 343 (2002).

[35] P. Y. Huang, S. Kurasch, A. Srivastava, V. Skakalova, J. Kotakoski, A. V. Krasheninnikov, R. Hovden, Q. Mao, J. C. Meyer, J. Smet, D. A. Muller, and U. Kaiser, Nano Lett. 12, 1081 (2012).

[36] M. Heyde, G. H. Simon, and L. Lichtenstein, Phys. Status Solidi B 250, 895 (2013).

[37] A. Alessi, S. Girard, M. Cannas, S. Agnello, A. Boukenter, and Y. Ouerdane, J. Light. Technol. 30, 1726 (2012).

[38] S. Ghemid, A. Monteil, and D. Guichaoua, Comput. Mater. Sci. 39, 552 (2007).

[39] G. Sastre and A. Corma, J. Phys. Chem. B 110, 17949 (2006).

[40] L.-G. Hwa, K.-J. Hsieh, and L.-C. Liu, Mater. Chem. Phys. 78, 105 (2003).

[41] D. B. Fraser, J. Appl. Phys. 39, 5868 (1968).

[42] K. Muralidharan, J. Torras, and S. B. Trickey, J. Phys. Condens. Matter 19, 386238 (2007).

[43] J. F. Shackelford and L. P. Davila, J. Non-Cryst. Solids 356, 2444 (2010).

[44] F. El-Diasty, Opt. Lasers Eng. 46, 291 (2008).

[45] U. Rabe, S. Amelio, E. Kester, V. Scherer, S. Hirsekorn, and W. Arnold, Ultrasonics 38, 430 (2000).

[46] D. C. Hurley, M. Kopycinska-Müller, A. B. Kos, and R. H. Geiss, Meas. Sci. Technol. 16, 2167 (2005).

[47] D. C. Hurley and J. A. Turner, J. Appl. Phys. 102, 033509 (2007).

[48] F. Mège, F. Volpi, and M. Verdier, Microelectron. Eng. 87, 416 (2010).

[49] G. Brambilla, F. Xu, P. Horak, Y. Jung, F. Koizumi, N. P. Sessions, E. Koukharenko, X. Feng, G. S. Murugan, J. S. Wilkinson, and D. J. Richardson, Adv. Opt. Photonics 1, 107 (2009). 